\title{
21 Morphology in Word Recognition
}

\author{
JAMES M. MCQUEEN AND \\ ANNE CUTLER
}

\section{Introduction}

This chapter reviews the psycholinguistic literature on the representation and processing of morphological structure in the recognition of spoken and written words. To anticipate more than a little, this is how we will conclude: psychological morphology does not map neatly on to linguistic morphology. We will argue that the processing and representation of morphologically complex words are determined by performance factors, including order of occurrence of stems and affixes, transparency, productivity and frequency of usage. For this reason, linguistic distinctions - for example, between derivational and inflectional relations - may not map neatly on to processing distinctions.

In psycholinguistic descriptions of the mental lexicon it is common to distinguish between access representations and central representations. Access representations are modality-specific processing structures involved in the mapping of visual or auditory input on to the lexicon. They provide the link between more peripheral levels of processing and the central lexicon, and they code form information (orthographic or phonological). Central representations are modality-independent structures coding words' meanings and their syntactic and thematic roles. We will be concerned with the role of morphology at both the access and the central levels.

There are two core issues to be addressed in this chapter. The first concerns the representation of morphological structure in the central lexicon. Is the mental lexicon organized in a way which codes morphological relationships? For example, is the fact that two words share the same stem, or the same affix, coded in the lexicon? This is primarily a question of representation: what information about the internal structure of words is stored in long-term memory, and how? But it is also a question of processing. When a word and its morphology are recognized, does this involve contact with other entries (words and/or morphemes) in the lexicon? 
The second issue is that of lexical access. What role does morphological structure play in the process of mapping perceptual information, from spoken or written input, on to the mental lexicon? Is morphological analysis necessary, optional or impossible prior to lexical access? Again, there are questions of both processing and representation: what type of morphological parsing might take place, and what form of access representations might be the product of such a process?

These two issues are in fact not independent. Underlying both is a question which is fundamental in many areas of psychology: the relative importance of rule-based processing and rote storage. Is it more 'effident' to hold in memory only that which cannot be derived by rule and to be dependent on the smooth operation of such rules, or to store every piece of information and to be dependent on efficient access procedures and the availability of storage space? In the case of morphology, this question becomes one of whether morphologically complex words are decomposed into their component stems and affixes before access to a central lexicon, in which lexical entries are considered to be shared by the morphological derivatives of each stem (Taft and Forster 1975), or whether each word form in a language has a separate, undecomposed entry in the mental lexicon, with no morphological analysis of any word form prior to lexical access (Butterworth 1983). The former of these alternatives assumes that all morphology is completely transparent to the word-recognition system, the latter that it is all opaque. Between these extremes, as the following sections show, fall numerous intermediate positions.

\section{Lexical representation of morphological structure}

\subsection{Are, morphological relationships represented?}

Words may be recognized faster if a morphologically related word has recently been processed; thus recognition of the 'targef pour in a lexical decision task (in which subjects judge whether a written or spoken item is a real word or not) is speeded by prior presentation of the 'prime' pours (Stanners et al. 1979a). This 'repetition priming' effect has been extremely influential in psycholinguistic studies of morphology. To be sure that it is a true morphological effect, however, one has to rule out alternative explanations invoking formal or semantic similarity. Car overlaps (both orthographically and phonologically) with card as much as it overlaps with cars; likewise, sameness and same have shared meaning, but to no greater extent than equivalence and same. A large body of research has been devoted to the question of whether morphological relationships are indeed represented independently of both formal and semantic relationships. 
One such line of research has demonstrated that morphological effects pattern differently from effects of formal similarity. For instance, orthographic priming is absent where morphological priming occurs, both in brief visual recognition following list learning (Murrell and Morton 1974) and in repetition priming tasks (Feldman and Moskovljevic 1987, Napps and Fowler 1987). Morphological priming is long-lived, while orthographic priming is very transient (cf. Napps 1989 with Napps and Fowler 1987). In the masked-priming paradigm, in which primes are presented very briefly and then immediately masked by the target, morphological priming is facilitatory, while orthographic priming is inhibitory (Drews and Zwitserlood 1995, Grainger et al. 1991). In unmasked lexical decision, in which the prime immediately precedes the target, orthographic priming is again inhibitory where morphological priming is facilitatory (Drews and Zwitserlood 1995, Henderson et al. 1984). Feldman and Moskovljevic (1987) alternated the two alphabets of Serbo-Croatian (Roman and Cyrillic), and showed an effect of morphology in repetition priming which was just as large when visual similarity was very low (prime and target in different alphabets) as when visual similarity was high (prime and target in the same alphabets). A similar result has been obtained with Hebrew (Feldman and Bentin 1994), in which, due to vowel infixation, words sharing the same root morpheme can have different orthographic forms. Feldman and Bentin found equivalent morphological priming when prime and target either had the same or different orthographic structures.

All of these results suggest that there is a level of representation of morphological information independent from the representation of orthographic information. As, for example, Drews and Zwitserlood (1995) have argued, one way of conceptualizing these separate levels is to distinguish between more peripheral access representations, where entries overlapping in form inhibit each other, and central representations, where morphological relationships are coded.

In the auditory modality, morphological priming occurs when phonological priming is absent. Kempley and Morton (1982), assessing priming of words spoken in noise, found effects of regular inflectional overlap (hedges - hedge) but not phonological overlap (pledge - hedge). Emmorey (1989), in an immediate priming task (50 milliseconds between prime and target), found priming between morphological relatives (e.g. submit-permit), but no priming between purely phonological relatives (e.g. balloon - saloon). These results again suggest that form information (here, phonological form) is represented independently of morphological information.

Semantic priming, like form-based priming, also appears to have a different time course to morphological priming. L. Henderson et al. (1984) showed priming for morphological relatives when the prime preceded the target by both 1 and 4 seconds, but priming of semantic relatives (synonyms) at the 1 -second interval only. Bentin and Feldman (1990) found semantic priming for written Hebrew with no intervening items between prime and target, but none with fifteen intervening items. When prime and target were morphologically related, 
there was priming at both lags, even when the prime and the target (derived from the same root) had only weak semantic association. Likewise, Emmorey (1989) showed priming between spoken words which were morphological relatives but not semantic associates (e.g. submit-permit). These results suggest that a model in which all morphological relationships in the lexicon are purely semantic (Butterworth 1983) is untenable. As Stolz and Feldman (1995) have argued, it appears that morphological effects cannot be reduced to either formbased effects (whether due to orthography or phonology) or effects of associative semantics.

\subsection{Models of representation}

How, then, is morphological information represented in the central lexicon? A number of alternative schemes have been proposed. One is that morphological relatives share a single lexical entry (e.g. Taft 1985,1988). In this framework all morphological derivatives of a stem are listed fully within the same entry, but in morphologically decomposed form (Taft 1988). A related, shared-entry model is the Augmented Addressed Morphology (AAM) model for inflectional morphology (Caramazza et al. 1988; see also Chialant and Caramazza 1995). In this model, entries consist of stems positively linked to the inflectional suffixes with which they can combine, and (for irregular verbs) of stems negatively linked to suffixes with which they cannot combine.

Alternative accounts make the assumption that words have separate entries. One of these assumes that morphological relatives are linked together as 'satellites' under a main entry, the 'nucleus' (Feldman and Fowler 1987; Gunther 1988; Lukatela et al. 1978, 1980; but see Kostic 1995 for problems with the satellite approach). Another type of separate-entries model envisages a network containing entries for words and, separately, for morphemes: lexical entries for morphologically related words are linked by a node which represents their shared-stem morpheme (S. Andrews 1986; Fowler et al. 1985; Grainger et al. 1991; Schreuder et al. 1990; Schriefers et al. 1991, 1992).

Schriefers et al. (1992), using the repetition-priming paradigm, provided evidence consistent with network models. Their study compared the size of priming effects between German inflectional and derivational forms, each form acting as both a prime and a target. The effects were asymmetrical: adjectival forms with the dative singular suffix $-e m$ primed suffixed relatives with $-e$ and -es and their stem forms, but -em forms were themselves not primed by any of these three types. In network models, each word is represented as a processing node; when a word has been presented, its node has a high degree of activation. Each stem morpheme also has a node associated with it. The word nodes of all the morphological relatives of a given stem have bi-directional facilitatory connections to the stem node. The basis of the priming effect is that activation of a word node in a morphological cluster boosts the activation of 
the stem node, and, indirectly, the activation of connected word nodes. On the assumption that the facilitatory connections can vary in strength (such that a word node can prime a stem node more or less than that stem node can prime either that or other word nodes), these priming asymmetries (and similar results of Feldman and Fowler 1987 and Feldman 1991) can be accounted for. It is not yet clear, however, which processing or linguistic factors may determine the relative strengths of these associative connections.

These results seem problematic for decompositional models such as that of Taft (1988), since in such models stem access should produce the same amount of priming irrespective of the form in which the stem occurred. They also seem problematic for satellite models. For Serbo-Croatian, Lukatela et al. (1980; see also Feldman and Fowler 1987) operationally defined nominative singular nouns (because they were responded to most rapidly) as the nuclei around which were clustered other nominal inflected forms. There was, however, no clear nucleus in the German adjectives studied by Schriefers et al. (no one form stood out as having faster response times associated with it).

Grainger et al. (1991) also interpreted their finding of facilitatory morphological priming and inhibitory orthographic priming in the masked priming task in terms of a network, suggesting that inhibitory connections might exist between visually similar words (morphologically related or not), with negative priming operating alongside positive morphological priming. Schreuder et al. (1990) examined the processing of Dutch verbs using a partial priming technique (Jarvella et al. 1987), in which part of a word is briefly displayed prior to presentation of the complete word (for naming). Priming of whole words by either affixes or stems was found only for verbs with separable particles. Since these particles can be separated from their stem by several words when the verb is inflected, and since the meanings of the complex forms are often not predictable from the joint meanings of particle and stem, there is good reason to suppose that both particle and stem have separate but closely linked representations. Again, Schreuder et al. account for their results in terms of a network model. More recent research on the processing and representation of separable verbs in Dutch, using a grammaticality judgement task, has provided further support for a network approach, where 'morphological integration' nodes provide the links between separate representations for particles and stems (Frazier et al. 1993).

Other results support lexical representation of morphological structure. For example, Tyler and Nagy (1990) found that readers made fewer semantic errors and more syntactic errors in selecting paraphrases of sentences with suffixed than with matched non-suffixed words. They argued that a morphologically structured lexicon would give the reader immediate access to morphological relatives of a suffixed form, increasing the likelihood of accessing the appropriate semantics (hence the low semantic error rate), but also increasing the likelihood of misidentifying a suffixed form as one of its relatives (hence the high syntactic error rate). Using eye fixation time data, Holmes and O'Regan (1992) found gaze durations for both prefixed and suffixed words to be shortest when the first fixation on a word included the stem. 
Laudanna et al. (1989, 1992) employed a stem homograph priming technique with Italian materials, in which pairs of words were presented (either simultaneously or sequentially) for lexical decision. Laudanna et al. (1989) showed that lexical decisions to pairs with homographic stems that were morphologically unrelated (e.g. portare 'to carry' and parte 'doors' with the stem port-) took longer than those to pairs with non-homographic stems (e.g. collo 'neck' and colpo 'blow' with the stems coll- and colp-), which in turn were slower than those to pairs where the stems were morphologically related (e.g. porta 'door' and porte 'doors'). Laudanna et al. (1992) replicated the inhibitory effect of orthographic overlap (relative to non-homographic controls) for word pairs sharing an inflectional stem (e.g. mute 'mute' and mutarano 'they changed' with the stem mut-), but did not find any effect for words sharing a homographic (but unrelated) derivational root (e.g. mute and mutevole 'changeable', where mutevole has the derivational root mut- but the inflectional stem mutevol-). Nevertheless, these authors also showed that derived words (e.g. mutevole) and inflected words (e.g. mutarano) were equally effective as primes for lexical decision on infinitival forms (e.g. mutare 'to change') in a priming experiment.

Laudanna et al. (1992) argue that words must therefore be represented in terms of their morphemic constituents, and that these constituents are inflectional stems and affixes, rather than derivational roots and affixes. They interpret their findings as evidence for their AAM model, in which lexical entries are morphologically decomposed (Caramazza et al. 1988). Lexical entries containing stems with the same orthographic structure (stem homographs) are considered to inhibit each other in order to resolve ambiguity. Morphological relatives facilitate each other through repeated access to the same stem. These results, however, can also be accommodated by network models, although they suggest that morpheme nodes should be based on inflectional stems rather than derivational roots, with inhibition between homographic stem nodes.

The data presented by Schriefers et al. (1992) also constrain network models. In addition to asymmetric inflectional priming, these authors obtained different patterns of priming for inflectional and derivational suffixes (of the same adjectival stems). Although not all priming effects were equal, both inflected and derived words primed, and were primed, by their stems, and inflected words primed other inflected words. However, there was no priming between derivational forms (e.g. Rote, rotlich). Feldman (1994) also compared inflectional and derivational priming in Serbian (the same language previously referred to as Serbo-Croatian). Although both inflectionally and derivationally related words produced facilitatory priming effects, those involving inflections were larger. The results for derivations thus contrast with the German findings. Further cross-linguistic experiments will be required before such differences can be explained. Nevertheless, it seems clear across several studies that inflectional priming is more robust than derivational priming.

Other results have indicated different patterns of priming dependent on the nature of the derivational relationship under test. Marslen-Wilson et al. (1994) used English materials in a cross-modal priming task (measuring lexical decision speed to written words, presented immediately after the offset of 


\section{James M. McQueen and Anne Cutler}

spoken word primes), and found that suffixed forms (e.g. friendly) primed and were primed by their stems, but that suffixed forms did not prime each other (e.g. confession, confessor). Derivationally prefixed forms (e.g. unfasten, refasten) primed and were primed by their stems, but, in contrast to suffixed forms, they also primed each other. Prefixed and suffixed forms sharing the same stem also primed each other (e.g. distrust, trustful).

Marslen-Wilson et al. argued that these results support a model in which lexical entries are morphologically decomposed, with affixes clustered around shared stem morphemes (their account is thus similar to the lexical component of Caramazza et al.'s (1988) AAM model). Priming takes place through repeated access to stems. All members of a cluster therefore prime each other. Suffixed forms do not prime each other, however, because additional inhibitory links between the suffixes in a cluster cancel out any benefit due to repeated access of the stem. Marslen-Wilson et al. justify these inhibitory connections on the grounds that during spoken word recognition, when the listener has only heard a stem, different suffixes of that stem are possible completions and should therefore all be activated. When evidence for one suffix arrives, the activation of incorrect suffixes needs to be suppressed - hence the inhibitory connections. No such connections are required between prefixes: a given prefix will not activate other prefixes of the same stem, so they need not be suppressed.

Although Marslen-Wilson et al.'s account is confined to derivational morphology, it suggests a more general way in which priming asymmetries (such as those reported for inflected words by Schriefers et al.) could be accommodated in shared-entry models (including their own model and Caramazza et al.'s AAM model). The basic claim would be that the shared entry does not consist of a simple listing of the affixes appropriate for a stem, but rather that the affixes are themselves structured processing units. In addition to the inhibitory connections postulated by Marslen-Wilson et al. for derivational suffixes, there could also be inhibition between inflectional suffixes. If the strengths of the connections between stems and affixes, and between affixes, were allowed to vary independently, then priming asymmetries between and within inflected and derived words could be explained. Such a model makes very similar predictions to those made by the network account proposed by Schriefers et al., where the strengths of connections between whole-word entries and their shared stems can vary.

An important constraint on the lexical representation of morphological information, both for network models and for internally structured sharedentry models, is that of semantic transparency. Marslen-Wilson et al. (1994) examined this issue explicitly. They asked subjects to rate the semantic relatedness of morphologically related word pairs, and found high estimates of relatedness for pairs such as confession - confessor, which they then defined as transparent, and low estimates for pairs such as successful-successor, which they defined as opaque. The effects of morphological structure described above could be obtained only when the morphological relationship between stem and affixed form was transparent; that is, friendly primed friend, but casualty did 
not prime casual. In other words, morphological relationships in the mental lexicon, however they may be represented, cannot be defined purely on formal linguistic grounds. Most studies of derivational morphology have failed to control for semantic transparency/opacity; some of the variability of morphological effects in the literature may result from this lack of control (as pointed out by L. Henderson 1985,1989, and by Marslen-Wilson et al. 1994). Note that although Emmorey (1989) did obtain priming between opaque forms (such as submit-permit), she employed a task in which both prime and target were presented auditorily (in contrast to Marslen-Wilson et al.'s cross-modal task). Furthermore, the morphological relationships between primes and targets tended to be less transparent in the Marslen-Wilson et al. study than in the Emmorey study.

Marslen-Wilson et al. also examined phonological transparency. They found as much priming in pairs such as elusive - elude and serenity - serene as in pairs such as friendly -friend. Fowler et al. (1985) and Downie et al. (1985) also found no effects of phonological transparency on the size of morphological priming effects. These results suggest that the locus of these effects is in the central lexicon, where representations have abstracted away from surface forms.

To summarize so far, the large priming literature indicates that morphological information is represented in the central lexicon. Recent research has shown that this includes detailed information on the relationships between the forms of a morphological family, more than just that they are related. This evidence therefore supports refined models of lexical organization: either those with separate entries for each word form which are linked with variable connections to entries representing their shared-stem morphemes (network models; Schriefers et al. 1992) or those with decomposed shared entries, with variable connections between stems and affixes, and between affixes (internally structured shared-entry models; Marslen-Wilson et al. 1994). As has often been pointed out (e.g. by Burani 1993), it can be difficult to distinguish between these alternative theoretical accounts.

It should be clear from the previous section that inflectional and derivational morphology could in principle be represented similarly in the lexicon, with semantic transparency of the morphological relationship being the main determinant of the strength of connections between related words. As Feldman (1994) has suggested, one reason why priming effects tend to be stronger for inflections than for derivations may be that inflectional relationships are in general more transparent than derivational relationships. There may be no need for a qualitative distinction in the way in which inflections and derivations are mentally represented.

Research on the representation of compounds leads to similar conclusions. Separate whole-word representations are often posited for compounds. Several authors (e.g. L. Henderson 1985; Sandra 1990,1994) have argued that since the meanings of nominal compounds such as blackbird are not fully recoverable from their components, they require independent meaning representations in the central lexicon. These whole-word representations are usually considered 
to be linked in a network to representations of their component morphemes (see e.g. network accounts offered to explain the storage of Chinese compounds: Taft and Zhu 1995, Zhou and Marslen-Wilson 1994). When novel nominal compounds are encountered, they appear to be interpreted via activation of the meanings of their constituents (Coolen et al. 1991, 1993).

As in the account of inflections and derivations, semantic transparency also plays a role in the representation of compounds. Sandra (1990) has argued on the basis of a study of Dutch compounds that although semantically opaque compounds (like blackbird) have independent central representations, fully transparent compounds may lack such representations, and may be recognized on the basis of activation of their constituent morphemes. Zwitserlood (1994), again from a study of Dutch compounds, has proposed a multiple-level lexicon. In addition to access (form) representations, she proposes two further levels: a morphological level, where the relationships between compounds and their component morphemes are coded, even for fully opaque compounds like klokhuis (lit. 'dock-house', but meaning core, as of an apple); and a semantic level, where fully opaque compounds are not connected to their constituents.

A structured central lexicon, with morphological relationships coded in an activation network, is therefore the favoured account of the representation of all complex words: inflected, derived and compound forms. It appears, however, to be unnecessary to posit qualitatively different representational accounts for these different classes. Factors such as semantic transparency, which apply to all classes, appear to determine the strength and nature of the connectivity between morphemes.

\section{Prelexical processing of morphological structure}

\subsection{Derivational morphology}

As we pointed out earlier, questions of the structure of central lexical representations are closely bound to questions of lexical access. What is the nature of the pre-lexical processes and the lexical access representations via which the central representations are contacted during word recognition? An influential paper by Taft and Forster (1975) argued for an obligatory process of pre-lexical decomposition, whereby words are broken down into their constituent morphemes prior to lexical access. This decomposition model (and its modifications - Taft and Forster 1976, Taft 1979a; see also Taft 1979b, 1981,1985,1988; Taft et al. 1986) proposes that all affixes are detected and stripped from a word before lexical access is attempted using the remaining stem morpheme. Revive, for example, would be accessed via its stem, vive. Taft and Forster (1975) based their proposal on non-word interference effects in a lexical decision task in English; non-words were rejected more slowly when they were bound stems 
(e.g. vive from revive) than when they were pseudo-stems (e.g. lish from relish). Subjects also found it more difficult to reject prefixed non-words with real stems (e.g. dejoice) than prefixed non-words containing no real stem (e.g. dejouse). This second interference effect has been demonstrated in both the visual and the auditory modalities (Taft et al. 1986). Taft (e.g. 1985,1988) proposed that stem morphemes are the access codes used in lexical lookup; in contrast to pseudo-stems, they cannot be rejected immediately because they succeed in making contact with a central lexical representation (but see also Taft 1994 for an alternative account based on interactive activation, in which pre-lexical prefix stripping is not required).

L. Henderson (1985) has argued, however, that data from processing of non-words may not reflect normal word recognition; rather, morphological decomposition of non-words may be attempted only when access based on whole-word representations fails to find a lexical entry. Experiments involving effects with real words avoid this problem. Consider a pair of words such as misplace and misery. Pre-lexical decomposition should, at least when these items are presented visually, strip mis from both words, delaying recognition of the pseudo-prefixed form misery (due to erroneous lookup of the false stem ery). Rubin et al. (1979) indeed found this pseudo-prefixation effect using a lexical decision task in English, but only when there were prefixed non-words in the experiment, not when there were no prefixed non-words. On the basis of this result, they argued that decomposition was only an optional strategy - not normally employed, but invoked by the presence of prefixed non-words. However, Taft (1981) has provided an explanation for the failure to find decomposition effects when prefixed non-words were absent: subjects were able simply to say 'yes' tos any item beginning with a prefix. Any effect of decomposition would thus be masked. Taft (1981) also observed a pseudo-prefixation effect in a naming task when there were neither non-words nor truly prefixed words in the experiment. In this situation, strategic decomposition would be impossible; subjects were nevertheless slower to initiate the pronunciation of pseudoprefixed words than non-prefixed words.

Bergman et al. (1988) also found pseudo-prefixation effects in lexical decision, in Dutch. L. Henderson et al. (1984), however, using a lexical decision task with a small proportion of potentially prefixed English words and non-words (thus avoiding the problem of strategic decomposition), found no difference in lexical decision latency between pseudo-prefixed words and monomorphemic control words; prefixed words were, if anything, responded to slightly more rapidly than both of the other word types.

Pseudo-affixation has also been studied with suffixes. Manelis and Tharp (1977) presented pairs of English suffixed forms (e.g. bulky - dusty) and pairs of pseudo-suffixed forms (e.g. fancy - nasty) in a lexical decision task in which subjects had to decide on the lexical status of both forms. They found no difference between these pairs. In addition, Henderson et al. (1984) and Bergman et al. (1988) both found no differences in lexical decision latencies for individually presented suffixed, pseudo-suffixed and monomorphemic control words. 
However, Manelis and Tharp (1977) also tested mixed pairs of words (i.e. one suffixed and one pseudo-suffixed, e.g. bulky - nasty) and found that such pairs were responded to more slowly than the unmixed pairs. When pseudosuffixed forms are paired with genuinely suffixed words, the processor may be misled into attempting their decomposition, increasing processing difficulty.

Bergman (1988) has argued that the fact that pseudo-prefixation effects are stronger than pseudo-suffixation effects can be accounted for by a left-to-right parsing process. If visual input is processed letter by letter, pseudo-prefixes will be recognized as prefixes, inducing a processing cost while the system recovers from its incorrect analysis. Pseudo-suffixes, on the other hand, are less problematic because they can be processed as a continuation of the stem.

Libben (1994) showed that lexical decisions to ambiguous novel compounds, like busheater (bus-heater or bush-eater) took longer than those to unambiguous compounds like larkeater. Libben argued that this result provided evidence for a decomposition process, and also suggested that parsing operates left-toright. As further evidence for a decomposition procedure, Libben (1993) has shown that subjects are slower reading aloud morphologically illegal nonsense words like rebirmity (re- only attaches to verbs, while -ity only attaches to adjectives) than legal nonsense words like rebirmize (where the selectional restrictions of the affixes are compatible). This latter finding is open to the same criticism as the older non-word studies, however; it does not indicate that morphological decomposition is a necessary pre-lexical procedure in the recognition of words.

Two further paradigms which have been used recently also suggest that readers can use morphological information in processing written material. In the first, the segment-shifting task (Feldman et al. 1995), subjects are required to shift an underlined portion of one word (e.g. harden) on to another word (e.g. bright), and then to say the resulting form (brighten). This task was found to be easier when the shifted portion was morphemic (e.g. harden) than when it was non-morphemic (garden). Similar results have been obtained in Hebrew, where affixes were infixed and no longer formed contiguous units (Feldman et al. 1995), and in Serbian (Feldman 1994). In the second paradigm (Beauvillain 1994), French subjects saw two French words, presented sequentially, and had to identify whether the second word was the same as the first. Parts of the words were presented in higher contrast. Judgements that the words were the same were faster when the high-contrast part corresponded to the stem of the word (e.g. reflux 'ebb') than when it did not (e.g. reflet 'reflection'). But again, although the data from both these tasks suggest that readers can take advantage of morphological information, they show neither that morphological parsing is obligatory nor that it is pre-lexical.

There is one particularly strong argument against mandatory pre-lexical decomposition of derived forms. An autonomous morphological parser, given an input which could be affixed, has to attempt decomposition on this form. For items which are not in fact morphologically complex, there will be a processing cost associated with recovery from the mis-parsing (this is the thinking 
behind the pseudo-affixation studies). Clearly, such a mechanism would be inefficient if the language it operated on contained a large number of pseudoaffixed forms. Schreuder and Baayen (1994) have shown that pseudo-prefixed forms occur frequently in both Dutch and English. In a corpus of Dutch text, around 30 per cent of words beginning with strings which could be prefixes were actually not prefixed, while the corresponding proportion for an English corpus was a staggering 80 per cent. Laudanna and Burani (1995) have shown that pseudo-prefixation rates in Italian are also very high. Baayen (1993) has shown, however, that high proportions of pseudo-suffixed forms do not occur.

These statistics suggest that mandatory pre-lexical decomposition of derivational prefixes would be highly inefficient. But they do not rule out decomposition completely. It may be the case that the recognition system is sensitive to the distributional properties of affixes. If so, decomposition may be more likely for prefixed forms where the orthographic string forming the prefix tends to occur only very rarely as a pseudo-prefix, and less likely when pseudoprefixation is common for that string. Laudanna et al. (1994) performed multiple regression analyses on lexical decision data from prefixed Italian non-words, and found support for this hypothesis. Laudanna and Burani (1995) have also shown that other factors, such as prefix length and affix productivity, may determine how derived forms are processed. There is a very important point here: experiments which treat all affixes as alike may fail to reveal either clear or accurate results (Laudanna and Burani 1995, Sandra 1994). Lack of sufficient control of affix types (and indeed stem types) may account for some of the variability of previous results; it is to be hoped that tighter controls will be adopted in the future.

The balance of the evidence indicates that lexical access of derived forms does not depend upon morphological decomposition, but that decomposition can occur. Except for the Taft et al. (1986) non-word study, however, all studies described so far in this section have assessed visual word recognition. In spoken word recognition, there is evidence that prefixed words are not decomposed. Tyler et al. (1988) compared recognition performance on English prefixed words and their free stems (e.g. amoral and moral). In a gating task, in which listeners were asked to identify successively longer stretches of a word, Tyler et al. (1988) showed that the prefixed forms could be confidently identified earlier than the stems. There was a similar advantage for prefixed items over stems in both lexical decision and naming tasks when response time was measured from word onset. All these results support models of word recognition in which incoming information is processed continuously; they do not support discontinuous decomposition models, which predict processing costs associated with prefixation. Schriefers et al. (1991), avoiding some potential methodological confounds in the Tyler et al. study, also found that prefixed Dutch words were recognized earlier than their stems in a gating task. They used a phoneme monitoring task as well. They reasoned that if listeners use lexical knowledge in detecting target phonemes, and if lexical access depends on decomposition into stems and affixes, than detection of a 
phoneme in a stem should be equally fast whether the stem occurs in isolation or in a complex form. Subjects in fact detected target phonemes faster in prefixed words than in stems, suggesting that decomposition does not occur in the processing of spoken prefixed words.

\subsection{Inflectional morphology}

The picture that is emerging from the analysis of derivational morphology is that, at least in the visual modality, pre-lexical decomposition is an optional process. It is a strategy available to the language user, but it does not play a mandatory role in normal word recognition. Furthermore, it may be more likely to occur for some prefixes than for others. Is the picture the same for inflectional morphology?

Non-word interference effects have been obtained for inflected Italian words (Caramazza et al. 1988). Non-words composed of verbal stems with inappropriate inflections (e.g. cantevi, in which the first-conjugation verb root cant- occurs with the second-conjugation suffix -evi) were harder to reject than non-words composed either of genuine stems with illegal suffixes (e.g. cantovi) or of illegal stems with genuine suffixes (e.g. canzevi). These in turn were harder to reject than non-decomposable forms made from illegal stems and affixes (e.g. canzovi). Caramazza et al. argued that Taft's decomposition model would predict cantovi and canzovi to be equivalent, since both have an illegal suffix which cannot be stripped pre-lexically. They claimed, in contrast, that pre-lexical decomposition is non-mandatory. In their Augmented Addressed Morphology model, lexical access is achieved by two procedures operating in cascade, one based on whole-word forms (for known words) and one based on morphemes (for novel words). Caramazza et al. assume that the morphemic access procedure exercises an effect only when the whole-word procedure fails (e.g. when it is presented with non-words containing legal morphemes). Thus their model claims that morphological decomposition is optional to the same degree for inflected as for derived words.

Caramazza et al.'s study, however, given its non-word materials, is open to the same criticism as other non-word interference studies. Studies with real words suggest a different picture. For example, Stanners et al.'s (1979a) repetition-priming studies showed differences between derived and inflected words. Regularly inflected verbs primed later decisions on their bases as much as the base verbs primed themselves. Irregularly inflected verbs (e.g. hung hang) and adjectival and nominal suffixed derivatives of verbs (e.g. selective select and appearance - appear) were less efficacious as primes for the verbs than the verbs themselves. Stanners et al. (1979a) interpreted their results as evidence of pre-lexical decomposition of regularly inflected forms. They argued that the weaker priming for derived and irregularly inflected forms was due to the representation of morphological structure in the lexicon, while the equivalence of repetition priming and regular inflectional priming indicated a pre-lexical 
morphological process. The regular inflectional suffix on a verb like pours could be stripped pre-lexically, and the verb would then be recognized via the access code pour. If repetition-priming reflects repeated use of access codes, then the regular inflectional priming effect would indeed be predicted to be as large as the identical repetition effect.

However, it now seems clear that there are components of the priming effect which are not due to normal processes of lexical access, but instead are due to episodic memory processes (remembering that a prime occurred while processing the target) or to strategies adopted by subjects in response to the demands of the experimental task (see e.g. Fowler et al. 1985, Monsell 1985, Napps 1989). It is necessary to control for these factors before we can interpret any morphological effects. Fowler et al. (1985) provided these controls. In order to reduce episodic effects, the lag between first and second presentations of the critical word pairs (regular form-base and derived form-base pairs with matched base-base pairs) was increased from an average of nine intervening items (as in Stanners et al.'s design) to forty-eight; practice effects were also controlled. Under these conditions, both inflectional and derivational priming were statistically equivalent to repetition priming. With a similarly long lag condition, Stanners et al. (1979b) also found priming from prefixed words to stems to be equivalent to repetition priming. Furthermore, Fowler et al. compared derived and inflected forms in both the visual and auditory modalities. They found very little difference in the size of the priming effects for regular and irregular forms (irregular items were orthographically and/or phonologically opaque when compared to their stems, including some suppletive past-tense forms of verbs). These findings appear to undermine the pre-lexical decomposition account of regular inflectional priming, because they indicate that the priming effect is insensitive to surface-form transparency (see also Downie et al. 1985). Since irregular forms are not open to surface-form decomposition, the locus of the priming effect cannot be pre-lexical. Instead, it must be lexical. This is the position adopted by Fowler et al. (1985): that priming effects are due to the morphological structuring of the lexicon. This view is consistent with the results on central lexical representation reported in section 2 .

A decomposition procedure for inflected forms predicts that under certain circumstances there may be a processing advantage for uninflected forms (but note that this is not a general processing advantage - overall, inflected forms are no more difficult to recognize than uninflected forms: Cutler 1983). Taft (1978) and McQueen et al. (1992) have shown that homophone pairs made up of a regularly inflected form and an uninflected form (e.g. billed-build) are more often recognized (written down in dictation) as the uninflected form, even when the inflected form is much more frequent (e.g. based-baste). This result suggests that the extra processing required in decomposition may delay recognition of the suffixed form. Jarvella and Meijers (1983) asked subjects to make same-different judgements on either the stems or the affixes of pairs of words. Subjects were raster to judge stems than inflections; Jarvella and Meijers argued that stem judgements were easier because they could be based 
on lexical representations of the stems, while inflection judgements were hard because they could not be based on independent representations of the inflected forms. Decomposition of inflected forms would prevent them having independent representations. It would also make them more difficult to remember: in a task where subjects had to recall lists of words, 17 per cent of the plurals in the lists were misremembered as their singular forms (Van der Molen and Morton 1979). Data from letter cancellation tasks is also consistent with the decomposition of inflected words; Smith and Sterling (1982; see also Drewnowski and Healy 1980) found that subjects were more likely to miss the letter $e$ in affixes or pseudo-affixes than in other syllables. The fact that the effect appeared in both affixes and pseudo-affixes suggests a pre-lexical procedure which would be blind to the true status of a possible affix. Finally, Gibson and Guinet (1971) presented written words very briefly for identification. Fewer errors were made on inflectional endings than on non-inflectional endings, suggesting that the suffix acted as a separate unit in perception. This result again favours decomposition of inflected words.

In summary, the evidence for decomposition reviewed so far is stronger for inflectional than for derivational forms. Decomposition may be an optional strategy for derived words, available when normal access procedures fail. It would therefore appear that derived words have independent whole-word access representations. Inflected words may not have their own access representations, and access to the central lexicon for inflected forms may be via decomposition, leading to access representations of their component stems and affixes.

\section{Frequency of occurrence}

We have already seen that processing factors appear to determine the role of morphological information in word recognition. At the level of representation in the central lexicon, factors such as semantic transparency influence which morphological relationships are represented and how they are coded. During pre-lexical processing and lexical access, factors such as the likelihood of letter strings appearing as genuine prefixes may determine whether or not decomposition of derived forms takes place. One important processing factor, the frequency of occurrence of words, has so far been overlooked. Word frequency is treated separately, since its analysis has had implications both for lexical access and for central lexical representations. This body of work exploits the well-established finding of 'frequency effects' - lexical decisions are faster to high- than to low-frequency words.

Taft (1979b) manipulated independently surface frequency (the frequency of occurrence of any particular surface form) and combined stem frequency (the summed frequency of occurrence of a stem across all the inflected forms in which that stem occurs). He found that lexical decisions to inflected and 
uninflected words were faster both for higher combined stem frequency (when surface frequency was controlled) and for higher surface frequency (when stem frequency was controlled).

Taft's materials included both nouns and verbs; other studies, however, have examined frequency effects for each syntactic class separately. Burani et al. (1984) replicated Taft's results with Italian regular verbs: both combined stem frequency and surface frequency predicted lexical decision time of inflected forms. However, Katz et al. (1991) found that only surface frequencies, not combined stem frequencies, could predict lexical decision time for uninflected English verbs. (Their results for inflected English verbs are ambiguous: decision latencies for past-tense forms were predicted by surface frequency alone, but those for present participles were predicted by both surface and combined stem frequency.) Sereno and Jongman (1992) found surface frequency effects for both inflected and uninflected verbs, but they observed no effect of combined stem frequency for uninflected verbs (combined stem frequency effects in inflected verbs were not tested).

Although effects of combined stem frequency are fairly reliable for inflected verbs, the evidence is somewhat contradictory for uninflected verb forms. A similar pattern emerges for nouns. Sereno and Jongman (in press) used a set of nouns matched on combined stem frequency but differing on the relative frequency of their uninflected and inflected forms, such that one subgroup had higher-frequency singular forms and lower-frequency plurals (e.g. river $(s)$ ), while the other group had higher-frequency plurals and lower-frequency singular forms (e.g. window(s)). Surface frequency effects in lexical decision latency were observed for plurals (e.g. windows faster than rivers). Although there was a similar effect for singulars (e.g. river faster than window), this effect was not significant. For nouns matched on surface frequency of their singulars but differing in combined stem frequency, no effect of combined stem frequency was observed in lexical decisions regarding the uninflected nouns, while there was a non-significant trend for the plurals (high combined frequency plurals were responded to somewhat faster than low combined frequency plurals). The failure to find surface and combined stem frequency effects for uninflected nouns appears to contradict Taft's (1979b) results.

The results for both nouns and verbs are therefore somewhat inconclusive. The picture is little clearer for derivational morphology. Taft (1979b) found that lexical decisions regarding prefixed words, matched in terms of their surface frequency, were faster for those with higher combined root frequency than for those with lower combined root frequency (the summed frequency of occurrence of a root across all the derived and inflected forms in which that root occurs). Extending Taff s result, Burani and Caramazza (1987) found surface and combined root frequency effects for Italian suffixed derived words.

But Burani and Caramazza's finding appears to conflict with one reported by Bradley (1980). She found no effects of surface frequency for English suffixed words ending in -ment, -er, -ness and -ion, and effects of combined root frequency only for the first three of these types of word (i.e. she found no frequency effects 
whatsoever for words ending in -ion). As Burani and Caramazza note, however, Bradley's word lists contained a high proportion of items with the same suffix. This could have induced a decompositional strategy, and hence reduced surface frequency effects. Strategic decomposition, since it would depend on the detection of decomposable forms, would also produce the apparent effect of transparency which Bradley obtained: -ion suffixes alter pronunciation, spelling and stress (and therefore by implication impair detectability) of their roots, while -ment, -er and -ness do not.

Cole et al. (1989), in support of Burani and Caramazza, have also observed surface and combined root frequency effects for French suffixed words. However, in contradiction of Taft (1979b), they found no effect of combined root frequency for prefixed words. S. Andrews (1986) observed a reliable frequency effect in bisyllabic compound words, such that those with a high-frequency word in their first syllable were responded to, in lexical decision, more rapidly than those with low-frequency first syllables (replicating Taft and Forster 1976). But she found that stem frequency influenced the recognition of bisyllabic suffixed words only in the context of compound words. Andrews argued that this was evidence for strategic rather than mandatory decomposition. Finally, in the eye-fixation study mentioned earlier (Holmes and O'Regan 1992), it was found that gaze durations for prefixed words matched on surface frequency were longer for those with low-frequency stems than for those with highfrequency stems.

Clearly, the evidence from all these studies of morphological frequency effects is ambiguous. Certainly, the fact that there are combined frequency effects at all further supports claims that morphological information is used during word recognition; but not only are there differences in the patterns of results obtained, there are also differences in the way in which these results have been interpreted. Taft (1979b) argued that combined stem frequency effects occurred because the stem acts as the access representation for all words with that stem. However, these results are also consistent with any model in which morphological information is represented in the central lexicon and in which lexical entries are frequency-sensitive. If representations of stems are linked to those for whole-word forms in a cluster, either within a shared entry or in a network, then recognition of any word in that cluster should be sensitive both to combined stem frequency and its own surface frequency. For example, Burani and Caramazza (1987) interpreted their findings as support for the AAM shared-entry model.

One finding does suggest that combined stem frequency effects are due to the central representation of morphology. Kelliher and Henderson (1990) found combined frequency effects for irregular past-tense inflected verb forms of English such as bought and shook, matched on surface frequency; responses were faster on those with higher-frequency infinitive forms (e.g. buy is more frequent than shake). As Kelliher and Henderson point out, it was impossible to determine whether this was indeed an effect of infinitive form frequency, of combined paradigm frequency (summing over both regular and irregular 
inflected forms) or even of combined root frequency, since these measures are very highly correlated. Nevertheless, the result suggests that the recognition of inflected verbs is influenced by the frequency of occurrence of their morphological relatives. Furthermore, the demonstration of this effect on the recognition of irregular past-tense forms, since by definition these do not share the same form as their stems, indicates that the morphological combined frequency effect has its locus in the central lexicon.

The clearest evidence of combined stem frequency effects was seen for inflected forms (both nouns and verbs). This is consistent with the evidence supporting pre-lexical decomposition of inflected forms, reviewed in section 3. If an inflected form is decomposed, it will be recognized via contact with an access representation and a central representation of its stem. Since all inflections for a given stem would be recognized in this way, the lexical representations (if frequency-sensitive) would show combined stem frequency sensitivity.

Considering all the evidence on decomposition, therefore, it would appear that neither extreme theoretical position (all complex words decomposed or no decomposition at all) is tenable. Dual-route models, where some words are morphologically parsed prior to access via their constituent morphemes and others are accessed directly via whole-word representations, appear best suited to account for the data. Dual-route models do not suffer from the criticism that decomposition is inefficient given high proportions of pseudo-prefixation, since in these models decomposition is not obligatory. On the other hand, dualroute models can account for the evidence that decomposition, at least under certain circumstances, does occur.

One dual-route model is the AAM model (Caramazza et al. 1988). As previously discussed, in the original formulation of the model the parsing route was slower than the whole-word route, and its behaviour could be observed only during the processing of forms composed of known morphemes but lacking in whole-word representations (novel words or non-words). In more recent formulations (Chialant and Caramazza 1995), parsing may also operate for complex words which occur rarely, but which have high-frequency constituents; but parsing is still considered to be slower than whole-word access.

An alternative dual-route account has been offered by Schreuder and Baayen (1995; see also Frauenfelder and Schreuder 1992). In this model, there are two routes which operate in parallel; they race with each other, such that whichever route finishes first will be responsible for lexical access on a given occasion. In general, the parsing route tends to be slower than the whole-word access route, since it involves more computational steps; but the two routes are assumed to vary in their processing times, with overlapping distributions in their completion times. Access representations are considered to be sensitive to combined stem frequency through a process of activation feedback. For some forms (low-frequency transparent inflections), there may be no wholeword representations, so their recognition can only be achieved via the parsing route. But high-frequency inflected forms are able to develop their own wholeword access representations. The development of whole-word representations 
depends both on frequency and on the complexity of the computations performed during parsing. Words of high frequency whose morphological parsing is complex (and hence more time-consuming) are the most likely to have their own access representations (thus speeding up their recognition).

Lexical decision experiments examining the recognition of singular and plural nouns varying in their relative frequencies in both Italian (Baayen et al. 1997a) and Dutch (Baayen et al. 1997b) support the detailed predictions of the dualroute race model and challenge those of the AAM model. Baayen et al. argue that the data from Italian suggest that storage of full-form representations does not occur for forms with singular suffixes (whose parsing is considered to be less complex), but can occur for forms with plural suffixes (whose parsing is considered to be more complex). But, in keeping with the race model, the evidence also suggested that not all plurals have whole-word access representations: Baayen et al. argue that those with higher frequency of occurrence are more likely to have their own representations.

Word frequency effects therefore appear to inform the debate on morphological processing, particularly on the nature of morphological parsing. Although results for derived forms are rather unclear, those for inflected forms combine with other evidence to suggest that at least some inflections may be decomposed prior to lexical access. Note again, however, that there need not be a qualitative distinction made between inflections and derivations. Dual-route models suggest that morphological decomposition is attempted on all complex forms, but it is only likely to be responsible for normal word recognition on a subset of forms, according to constraints such as transparency, productivity and word frequency (low-frequency transparent forms involving productive affixes, such as noun plurals, may be the most likely candidates for normal parsing - a word such as napes may well be recognizable only via decomposition).

One final cautionary remark needs to be made about word frequency effects. Schreuder and Baayen (1997), in a study of the recognition of monomorphemic Dutch nouns, have found that at least in tasks requiring higher-level processing of visually presented words, the size of the morphological family of the noun (the number of different derived or compounded words containing the noun as a constituent) influenced performance. Nouns were responded to more rapidly in lexical decision if they came from larger families, and they elicited higher subjective familiarity ratings. There was no effect, however, of family frequency (the combined frequency of all members of the complete morphological family). The lack of control of family size may account for some of the previous variability in morphological frequency effects.

\section{Conclusion}

The evidence we have reviewed leaves no doubt that morphological information is represented in the mental lexicon in a quite detailed way. Moreover, the 
most recent evidence suggests that there is variability between the strengths of the connections between different members of a morphological family. Models which state that morphological relatives are simply linked are not sufficient. Network models (e.g. Schriefers et al. 1992) and internally structured sharedentry models (Caramazza et al. 1988, Chialant and Caramazza 1995, MarslenWilson et al. 1994) can both deal with the variability requirement. Access to morphologically structured lexical representations, on the other hand, need not involve morphological structure overtly; decomposition of derived forms may be an optional procedure, available when the normal whole-word access procedure fails. Here the evidence differs according to type of morphological relationship: regular inflectional forms may be decomposed, perhaps depending on their frequency of occurrence, but derived forms probably are not.

Of course, lexical representation and lexical access are not independent; they form part of a unified word recognition system. This system, we would argue, is structured for the processing of spoken language. (Thus it is unfortunate that so large a proportion of studies of morphology in recognition, like studies in many other areas of Psycholinguistics, have been based on written materials.) As described above, Tyler et al. (1988) and Schriefers et al. (1991) have argued that lexical access of spoken words is a continuous process from the beginning of the word's presentation. In accounting for differences in priming between prefixes and suffixes, Marslen-Wilson et al. (1994) depend on this beginningto-end assumption. The temporal nature of the speech input also forms the basis for Cutler et al.'s (1985) explanation of the bias towards suffix morphology in the world's languages; listeners prefer to process stems before affixes. Arguments have also been made for beginning-to-end processing in visual word recognition (Bergman 1988, Cole et al. 1989, Hudson and Buijs 1995).

Both network and decomposed shared-entry models are compatible with temporally continuous access. For network models, there are separate entries for each word form, so access would be direct to each entry. Effectively, any stem has multiple representations, one for each inflectional variant (in addition to its representation in the stem-morpheme node binding the entries together). Shared-entry models economize by representing each stem only once. Access for prefixed words would begin with contact being made with a representation of the prefix, and access of suffixed words would begin at a representation of the stem. But in both cases, decomposition would take place as more information arrived in a temporally continuous manner.

Given that the vast majority of inflections are suffixes, temporally continuous access is obviously consistent with the decomposition of inflected forms suggested by the evidence reviewed in sections 3 and 4 . Initially, the stem portion of a word is mapped on to its lexical representation. When the suffix arrives, it can be mapped on to the representation of the inflected form. Suffixed words are therefore accessed via their stem. Decomposition is not delayed until contact has been made with a full form in the lexicon; it is achieved before the form has been identified. In this sense, decomposition takes place before word recognition. But it takes place during lexical access, not prior to it. 
Temporally continuous processing has further implications. One is that it may underlie observed differences between prefixed and suffixed forms. Cole et al. (1989) argued that combined root frequency effects were detectable on suffixed but not prefixed words, because recognition of prefixed forms does not initially entail access to the stem. Root frequency thus has less opportunity to influence recognition time in prefixed words than in suffixed words. Another implication is that not only suffixed inflectional forms, but also suffixed derived forms - as long as they are sufficiently transparent - should be decomposed during lexical access. In other words, processing distinctions between inflectional and derivational morphology arise not from a categorical distinction between these types, but strictly from processing considerations: the relative ordering of stem and affix and the relative transparency of the relationships involved. Note that Marslen-Wilson et al.'s (1994) results suggest that it is semantic transparency, rather than phonological (or, by extension, orthographic) transparency, which determines whether a morphological relationship is lexically represented. This suggests that lexical representations are abstract: that they represent the underlying phonological structure of a morphological family, rather than each individual surface form.

A related factor which may determine morphological representation is productivity. Forms with highly productive affixes are more likely to be semantically transparent, and hence represented in a decomposed way. Badecker and Caramazza (MORPHOLOGY AND APHASIA) describe patients who show morphological errors (in production) on inflections and productive derivations but not on non-productive derivations. Finally, frequency of usage may determine the strength of connections between suffixes and their stems; for example, Schriefers et al. (1992) found the weakest priming for the low-frequency adjectival suffix - em.

The most important characteristic of the above list is that order of occurrence, transparency, productivity and frequency are all essentially performance factors. Processing factors, we would argue, underlie both our general conclusions: that lexical representations are morphologically structured, and that lexical access involves decomposition of some morphologically complex words but not of others. Clearly, there are likely to be differences across languages in the extent to which morphological structure is lexically represented. Here, again, it is particularly unfortunate that most of the evidence available is from English and other closely related languages. Speakers of languages with very transparent, productive or simple morphologies may have a much richer mental morphology than speakers of complex, opaque languages. Hankamer (1989) has convincingly argued for the necessity of morphological parsing in agglutinative languages such as Turkish (but see also Niemi et al. 1994 for related arguments concerning Finnish, another language with rich combinatorial morphology). Such cross-linguistic differences will be motivated, however, not by the nature of the morphological systems themselves, but rather by the processing considerations which determine the structure of the human word recognition system. 


\section{ACKNOWLEDGEMENTS}

Much of the preparation of this chapter was carried out when both authors were at the MRC Applied Psychology Unit, Cambridge, where the first author was supported by the Joint Councils Initiative in Cognitive Science and HCI grant no. E304/148. We would like to thank Harald Baayen and Rob Schreuder for their comments on a previous version of this chapter. 